\title{
Sex Differences in Cardiovascular Risk Profiles of Ischemic Stroke Patients with Diabetes in the Greater
} Cincinnati/ Northern Kentucky Stroke Study

\section{Running Title: Sex Differences in Diabetes and Stroke}

Authors:

\author{
Tracy E. MADSEN, MD, ScM*1 \\ Jane C. KHOURY, PhD ${ }^{2}$ \\ Kathleen A. ALWELL, RN, BSN ${ }^{3}$ \\ Charles J. MOOMAW, $\mathrm{PhD}^{3}$ \\ Stacie L. DEMEL, DO, $\mathrm{PhD}^{4}$ \\ Matthew L. FLAHERTY, MD ${ }^{3}$ \\ Daniel WOO, MD, MS ${ }^{3}$ \\ Jason MACKEY, MD ${ }^{5}$ \\ Felipe DE LOS RIOS LA ROSA, MD ${ }^{3,6}$ \\ Sharyl MARTINI, MD, PhD ${ }^{7}$ \\ Simona FERIOLI, MD ${ }^{3}$ \\ Opeolu ADEOYE, MD, $\mathrm{MS}^{3}$ \\ Pooja KHATRI, MD, MSc ${ }^{3}$ \\ Brett M. KISSELA, MD, $\mathrm{MS}^{3}$ \\ Dawn KLEINDORFER, MD $^{3}$
}

1: Division of Sex and Gender in Emergency Medicine

Department of Emergency Medicine

The Alpert Medical School of Brown University

Providence, RI

2: Cincinnati Children's Hospital Medical Center, Cincinnati, $\mathrm{OH}$

3: University of Cincinnati College of Medicine, Cincinnati, $\mathrm{OH}$

4: Michigan State University, East Lansing, MI

5: Indiana University, Indianapolis, IN

6: Baptist Health Neuroscience Center, Miami, FL

7: Michael E. DeBakey VA Medical Center, Houston, TX

*Corresponding Author

55 Claverick Street, $2^{\text {nd }}$ floor

401-489-5275

401-444-6662

Tracy_Madsen@brown.edu

Madsen, T. E., Khoury, J. C., Alwell, K. A., Moomaw, C. J., Demel, S. L., Flaherty, M. L., Woo, D., Mackey, J., De Los Rios La Rosa, F., Martini, S., Ferioli, S., Adeoye, O., Khatri, P., Kissela, B. M. and Kleindorfer, D. (), Sex Differences in Cardiovascular Risk Profiles of Ischemic Stroke Patients with Diabetes in the Greater Cincinnati/ Northern Kentucky Stroke Study. Journal of Diabetes. Accepted Author Manuscript.

http://dx.doi.org/10.1111/1753-0407.12567 
Background:

We aimed to compare sex-specific associations between cardiovascular risk factors and diabetes mellitus (DM) among patients with acute ischemic stroke (AIS) in the Greater Cincinnati/Northern Kentucky Stroke Study (GCNKSS).

Methods:

The GCNKSS ascertained AIS cases in 2005 and 2010 among adult (age $\geq 20$ years) residents of a biracial population of 1.3 million. Past and current stroke risk factors were compared between those with and without DM using chi-square and multiple logistic regression to examine sex-specific profiles. Results:

There were 3515 patients with incident AIS; 1919 (55\%) were female, 697 (20\%) were black, and 1146 (33\%) had DM. Among both women and men with DM, significantly more were obese, and had hypertension, high cholesterol, and coronary artery disease (CAD) compared with those without DM. For women with AIS, in multivariable sex-specific adjusted analyses, older age was associated with a decreased odds of having $\mathrm{DM}(\mathrm{aOR}=0.88,95 \% \mathrm{Cl} 0.80-0.98)$. For women with $\mathrm{CAD}$, the odds of DM were increased $(a O R=1.76,95 \% \mathrm{Cl} 1.33-2.32)$. Age and $C A D$ were not significant factors in differentiating the profiles of men with and without DM.

Conclusions:

Women with DM had strokes at a younger age, whereas no such age difference existed in men. As opposed to men, women with DM were also more likely to have CAD compared with those without DM, suggesting a sex difference in the association between DM and vascular disease. These findings may suggest a need for more aggressive risk factor management in diabetic women.

Keywords: stroke, diabetes, sex differences, risk factors

Highlights:

This article is protected by copyright. All rights reserved. 
Among patients with incident ischemic stroke in the Greater Cincinnati Northern Kentucky Stroke Study, younger women and women with coronary artery disease (CAD) were more likely to have diabetes, whereas these associations were not significant for men. Our findings suggest that there is a sex-specific association between younger age and incident strokes in women with diabetes; as a consequence, women with diabetes may benefit from more aggressive risk factor control.

\section{Introduction:}

Diabetes mellitus (DM) is a major risk factor for ischemic stroke; risk of stroke is 2 to 6 times higher for those with diabetes compared with those without diabetes and increases with the duration of diabetes. ${ }^{1-5}$ In addition, data suggest that the overall prevalence of DM, as well as the prevalence of DM in stroke patients, has been increasing over time and will continue to rise. ${ }^{2,6}$

For women in particular, DM has been classified as a risk factor equivalent to prior stroke in non-diabetics. ${ }^{7}$ Previous analyses comparing women and men directly have shown that the risk of incident ischemic stroke among patients with DM is greater in women than in men, ${ }^{8}$ and sex-stratified analyses have reported a higher incidence of ischemic stroke among diabetics in women compared with men overall and over time. ${ }^{9}$ This parallels results that have shown that diabetes is a stronger risk factor for coronary artery disease (CAD) for women compared with men. ${ }^{10,11} \mathrm{~A}$ meta-analysis that pooled data from 64 cohorts and reported sex-specific estimates of the relative risk (RR) of ischemic stroke in those with DM compared with those without DM reported a RR of 2.28 for women, compared with 1.83 for men. The risk of stroke in this study for diabetic women was $27 \%$ higher than for diabetic men when direct sex comparisons were performed. ${ }^{8}$ Possible factors that may lead to sex differences in the risk of ischemic stroke among diabetics include differences in the treatment or severity of DM, sex differences in the mechanism by which DM leads to cerebrovascular disease, or a sex difference in the risk factor This article is protected by copyright. All rights reserved. 
profile between women and men. For example, it may be that women with DM have an overall worse risk factor profile than men with DM.

Objective

Our study objective was to compare sex-specific associations between cardiovascular risk factors and diabetes mellitus (DM) among patients with acute ischemic stroke (AIS) in the Greater Cincinnati/Northern Kentucky Stroke Study (GCNKSS).

\section{Methods:}

Study Population

This is a cross-sectional study of patients with incident ischemic stroke in the Greater Cincinnati Northern Kentucky Stroke Study (GCNKSS). The GCNKSS is a population-based dataset comprised of stroke patients from a biracial population of approximately 1.3 million people residing in a 5-county region of southern Ohio and northern Kentucky. This biracial population is representative of the United States population in terms of age, percent of people that are black, household income, level of education completed, and proportion of people below the poverty line. Cases of stroke among residents of these 5 counties are ascertained for a full calendar year every 5 years. Potential stroke cases are identified using discharge ICD-9 codes 430-436 from all acute-care hospitals in the study region. Data for each case is abstracted by study nurses and then reviewed by trained study physicians using a combination of clinical and imaging data to verify whether the case represents an acute stroke. Cases of stroke are also ascertained from local outpatient clinics, from county coroner data, and from a random sample of primary care doctors and nursing homes. Further details of the GCKNSS methodologies, including case definition and ascertainment have been previously published. ${ }^{12,13}$ For the present study, only adults (age of 20 years or greater) with incident ischemic stroke occurring in calendar years 2005 or 2010 were included for analysis. The two time periods (2005 and 2010) are cross-sectional incident populations and were analyzed together. Ascertainment methods and case definition were consistent

This article is protected by copyright. All rights reserved. 
over the two time periods. The institutional review board at each participating hospital approved the study.

\section{Data collection}

Data regarding patients' demographic characteristics (age and race) as well as history of DM and cardiovascular risk factors were obtained from patient medical records by trained study nurses. In order to be categorized as having DM, there must have been documentation of DM as a medical problem prior to the incident stroke event. Patients with undiagnosed diabetes were categorized as not having DM for the purposes of this study. Risk factors obtained from documented medical history in patients' charts included prior diagnoses of hypertension, high cholesterol, coronary artery disease (CAD), myocardial infarction (MI), atrial fibrillation, and current smoking status. For hypertension, patients had to have a documented medical history of hypertension to be counted in this category. Elevated cholesterol was defined as a prior history of elevated total cholesterol or LDL; terms including hyperlipidemia, hypercholesterolemia, and dyslipidemia were included in this definition. History of CAD was defined as prior myocardial infarct, $\mathrm{CABG}$, coronary angioplasty/stenting, or unstable angina. Atrial fibrillation was defined as having been diagnosed with atrial fibrillation in the past or being in atrial fibrillation on admission. Current smoker was defined as any cigarette smoking in the last 3 months. Height and weight were abstracted from the patient chart to calculate BMI. In addition to analysis as a continuous variable, obesity was treated as a binary variable, with obese defined as $\mathrm{BMI} \geq 30 \mathrm{~kg} / \mathrm{m}^{2}$." Reported medications prior to the stroke event were also abstracted from patient charts and categorized into lipid lowering medications, anti-hypertensive medications, anticoagulants, and aspirin (ASA)/ antiplatelet agents.

\section{Statistical analysis}

First, the study population was described in terms of baseline characteristics: age, black race, and history of DM. These characteristics were compared between women and men using t-test for age and chi-square for black race and history of diabetes. Bivariate analyses were used to compare mean

This article is protected by copyright. All rights reserved. 
age, the proportion of patients with age $>65$ years, body mass index $(\mathrm{BMI}), \mathrm{BMI} \geq 30 \mathrm{~kg} / \mathrm{m}^{2}$, and each of the pre-specified cardiovascular risk factors in sex-specific analyses (women with DM compared with women without DM, men with DM compared with men without DM). The risk factors including history of hypertension, high cholesterol, CAD, myocardial infarction, atrial fibrillation, and current smoker vs. nonsmoker/former smoker were treated as binary variables. T-tests were used for the continuous variables of age and $\mathrm{BMI}$, and chi-square tests were performed for the categorical variables, and associated $\mathrm{p}$-values were reported. Bonferroni corrections were applied due to multiple comparisons. Descriptive statistics (means and standard deviations or frequencies and proportions, as appropriate) were reported.

Bivariate analyses were also used to compare medications reported on admission. Only medications listed as being taken prior to the stroke event were included. For each category of medications (lipid lowering medications, antihypertensive medications, ASA/ antiplatelet agents, and anticoagulants), proportions of women versus men were compared in each study period $(2005,2010)$. Frequencies, proportions, and p-values were reported.

Following bivariate analyses, logistic regression was performed by sex to examine the sexspecific odds of a patient with specific pre-defined cardiovascular risk factors having a pre-existing diagnosis of DM. The odds for each independent risk factor were adjusted for all other risk factors to account for potential clustering. Adjusted odds ratios (aOR) are reported. Further analyses involved a logistic regression including both women and men with diabetes as the dependent variable and the interaction of sex with each risk factor examined. Discriminant analysis was used to examine the clustering of the risk factors within the sex or diabetes groups.

In all analyses, $p$-values $<0.05$ were considered to be statistically significant. All analyses were performed using $\mathrm{SAS}^{\circledR}$ version 9.4 (SAS Institute, Cary, NC).

This article is protected by copyright. All rights reserved. 


\section{Results:}

In total, 3515 patients with incident ischemic stroke were included in the analysis; 1919 (54.6\%) were female, 697 (19.8\%) were black, and 1146 (32.6\%) had histories of DM. Women were older than men $(72.0 \pm 15.0$ years vs. $67.2 \pm 14.0$ years, $p<0.0001)$. Similar proportions of women and men were black (compared with white) ( $n=395,20.6 \%$, vs. $n=302,18.9 \%, p=0.22)$, and a similar proportion of men and women had a history of DM ( $n=627,32.7 \%$, vs. $n=519,32.5 \%, p=0.92)$.

In unadjusted analyses (Table 1), higher proportions of women with DM were obese, black, and had hypertension, high cholesterol, and CAD, compared with women without DM. In similar comparisons, among men with DM, more were obese, had hypertension, high cholesterol, and CAD than men without DM. Proportions of patients with atrial fibrillation were similar between those with and without DM in both men and women. Women with DM were younger, on average, than women without DM (mean $70.7 \pm 13.4$ vs. $72.7 \pm 15.7$ years, $p=0.006$ ), whereas men with and without $D M$ were similar in age (67.1 \pm 13.1 years vs. $67.2 \pm 14.5$ years, $p=0.87)$.

In direct comparisons of risk factors between women with DM and men with DM, higher proportions of women were obese ( $48.7 \%$ vs. $42.7 \%, p=0.05)$ or black $(28.6 \%$ vs. $22.4 \%, p=0.02)$ compared with men. Similar proportions of women and men had hypertension $(91.6 \%$ vs. $89.4 \%$, $p=0.22)$, and a slightly higher proportion of men had CAD ( $44.7 \%$ vs. $40.4 \%, p=0.14)$ compared with women.

In adjusted analyses (Table 2), among ischemic stroke patients, for women, increasing age decreased the odds of having a pre-existing diagnosis of diabetes (aOR $0.88,95 \% \mathrm{Cl} 0.80-0.98$ for each 10 year increase), whereas for men age was not a significant factor (aOR $0.99,95 \% \mathrm{Cl} 0.89-1.10$ for each 10 year increase). Another sex difference was in the odds of having DM among those with CAD: women with CAD were more likely to have had a pre-existing diagnosis of DM (aOR 1.80, 95\%Cl 1.36-2.38), but men with CAD had similar odds of having had DM compared with those without CAD (aOR 1.26, 95\%Cl

This article is protected by copyright. All rights reserved. 
0.94-1.68). When interaction terms were included in the model with diabetes as the dependent variable, the interaction term between sex and age had a p-value of 0.07 , and the interaction term between sex and CAD had a p-value of 0.01 . There were significant positive associations between BMI, black race, hypertension, high cholesterol and our dependent variable of DM in both women and men. Both men and women with $\mathrm{MI}$ and atrial fibrillation had similar odds of having DM compared with men and women without these risk factors. Finally, both men and women who were current smokers had lower odds of having DM compared with men and women who were not current smokers. In both sexes, the associations between hypertension or obesity and DM were higher than the associations between other cardiovascular risk factors and DM.

Examination using discriminant analysis showed that about $70 \%$ of the patients could be correctly categorized as having diabetes versus not having diabetes within the sex-specific groups using the chosen risk factors. This is consistent with the c-statistics from the multiple logistic regressions of 0.74 and 0.71 for women and men, respectively.

With regard to medications on admission (Table 3), similar proportions of women and men were on anticoagulant medications in both study periods. More women than men were on ASA or other antiplatelet agents in 2005, but there was no difference by sex in 2010. More women were on antihypertensives in both study periods. Finally, a larger proportion of men were on medications for hyperlipidemia in 2005, but proportions were similar between women and men in 2010.

\section{Discussion:}

Previous literature has reported sex differences in the association between DM and incident stroke; ${ }^{8,9}$ however, our study is novel in its sex-specific description of the cardiovascular risk profiles of men and women with DM among ischemic stroke patients. Though there were many similarities in the risk factors associated with DM in women compared with men, we did find some sex-specific differences in demographics and risk factors between patients with DM and those without DM: women with a pre-

This article is protected by copyright. All rights reserved. 
existing diagnosis of DM were younger and more likely to have CAD compared with those without a diagnosis of DM, but differences were not significant for these risk factors in men.

The younger age of women with DM suggests that in women, DM may lead to more rapid progression of cerebrovascular disease that ultimately results in stroke at younger ages. Though presumably DM leads to stroke earlier in life in both sexes, there was no significant age difference between men with and without DM in our study, suggesting that this may not be true to the same degree in men compared with women. It is possible that there are risk factors that are more important than DM for men with regard to stroke risk. The difference in mean ages between women with and without DM was about 3 years; if women with DM have strokes 3 years earlier than women without DM, this has the potential to lead to more years of long-term disability and higher societal cost. Reasons for this age difference are not clear, but one could theorize that there are differences in the treatment of DM or that changes in exposure to sex hormones over the lifespan affect the mechanism by which DM leads to vascular dysfunction and stroke. ${ }^{14}$ Future efforts should examine whether women with DM may warrant more aggressive control of their DM and/or of other cardiovascular risk factors in order to prevent stroke. Tight glycemic control has not clearly been shown to reduce stroke risk, ${ }^{1}$ but future research should examine whether such an approach could potentially be more effective for women than men.

These data are also interesting in light of our previous analyses ${ }^{2,3}$ that conclude that the risk of stroke conferred by DM is greatest in patients less than 65 years and that ischemic stroke patients with DM are younger than those without DM. Based on the present study, this may be largely driven by women with DM. Data showing that women with DM were more likely to have CAD than women without DM is consistent with prior data showing that DM carries a greater risk of cardiovascular outcomes in women compared with men. ${ }^{10}$

This article is protected by copyright. All rights reserved. 
Of note, in both women and men, the odds of DM among current smokers were lower than in non-smokers or former smokers. This conflicts with known evidence that smoking is a risk factor for diabetes. ${ }^{15}$ Reasons for our findings are unclear and could be confounded by the inclusion of patients with type 1 diabetes, as smoking is primarily a risk factor for type 2 diabetes. ${ }^{15}$ Interventions to promote smoking cessations should continue to be a priority among patients at risk for stroke.

Our results regarding the high prevalence of hypertension, obesity, and high cholesterol in the stroke patients in our study also suggest a need for more effective interventions in both sexes. Since obesity is an important risk factor for both recurrent vascular events as well as other health outcomes, this result suggests that obesity should continue to be a major target for intervention among stroke patients, especially women. Unfortunately, some trials of lifestyle interventions to reduce obesity have been less effective in women. ${ }^{16}$ In addition, when patients with and without DM were compared (both men and women), the associations between hypertension, obesity, and high cholesterol and DM were higher than associations for other risk factors including age, smoking status, atrial fibrillation, and history of myocardial infarction. Consistent with prior data, this suggests that among stroke patients, there is often clustering of DM with the additional risk factors ${ }^{9,17,18}$ that comprise metabolic syndrome. ${ }^{19}$ Our study has some limitations. Because we did not include patients with undiagnosed DM, we may have underestimated the prevalence of DM. There may also be sex differences in undiagnosed DM of which we are not aware. In addition, we were not able to separate patients with insulin-dependent diabetes from those with non-insulin dependent diabetes, and these patients may have important distinctions in their risk factor profiles. ${ }^{18}$ Finally, we were not able to assess whether there were sex differences in the level of control of DM cardiovascular risk factors including levels of hypertension, cholesterol, and triglycerides. ${ }^{10}$ Future studies should investigate potential sex differences in treatment, differences in disease severity, and duration of disease for DM and other risk factors.

This article is protected by copyright. All rights reserved. 
Our study of sex differences in the demographics and risk factor profiles of stroke patients with DM adds to the current knowledge around stroke prevention strategies in women with DM, but further research is needed to better understand the relationship between DM and stroke in women. ${ }^{16}$ For example, it is unknown whether more aggressive control of DM and other cardiovascular risk factors like obesity and hypertension might benefit women by slowing down the progression of cerebrovascular disease and mediating the risk of stroke from DM. This is especially important given previous data showing women are less likely to meet blood pressure goals and that hypertensive diabetics are at an especially high risk for a primary cerebrovascular event. ${ }^{1,10,16}$ These data also reinforce the clustering of metabolic risk factors among patients with DM. In addition, future research should examine whether there are sex differences in the association between risk of recurrent stroke and DM as this could lead to sex-specific secondary stroke prevention strategies targeted at the treatment of DM and related vascular risk factors. A better understanding of both the overall and sex-specific risk factor profiles of patients with ischemic stroke and DM could lead to more effective and personalized primary and secondary stroke prevention strategies in diabetic patients.

\section{Acknowledgments:}

The GCNKSS is supported by funding from the NIH NINDS (R01NS30678).

Disclosures: Jane Khoury, Kathleen Alwell, Charles Moomaw, Matthew Flaherty, Daniel Woo, Jason Mackey, Simona Ferioli, Pooja Khatri, Brett Kissela, and Dawn Kleindorfer are supported by a research grant from the NINDS (R01NS30678). Matthew Flaherty is on the speaker's bureau for Janssen and CSL Behring and is a consultant for Portola. Pooja Khatri is supported by Genentech (research support) and Medpace (research support), is a consultant for St. Jude's device development and Grand Rounds Experts, and receives support from uptodate.com.

This article is protected by copyright. All rights reserved. 


\section{References:}

1. Goldstein LB, Bushnell CD, Adams RJ, et al. Guidelines for the Primary Prevention of Stroke: A Guideline for Healthcare Professionals From the American Heart Association/American Stroke Association. Stroke. 2011; 42: 517-584.

2. Khoury JC, Kleindorfer D, Alwell K, et al. Diabetes mellitus: A risk factor for ischemic stroke in a large biracial population. Stroke. 2013; 44: 1500-1504.

3. Kissela BM, Khoury J, Kleindorfer D, et al. Epidemiology of ischemic stroke in patients with diabetes: The greater Cincinnati/Northern Kentucky stroke study. Diabetes Care. 2005; 28: 355359.

4. Huang ES, Laiteerapong N, Liu JY, John PM, Moffet HH, Karter AJ. Rates of complications and mortality in older patients with diabetes mellitus: the diabetes and aging study. JAMA Intern Med. 2014; 174: 251-258.

5. Staszewsky L, Cortesi L, Baviera M, et al. Diabetes mellitus as risk factor for atrial fibrillation hospitalization: Incidence and outcomes over nine years in a region of Northern Italy. Diabetes Res Clin Pract. 2015; 109: 476-484.

6. Wild S, Roglic G, Green A, Sicree R, Hilary K. Global Prevalence of Diabetes: Estimates for the year

This article is protected by copyright. All rights reserved. 
2000 and projection for 2030. Diabetes Care. 2004; 27: 1047-1053.

7. Ho JE, Paultre F, Mosca L. Is Diabetes Mellitus a Cardiovascular Disease Risk Equivalent for Fatal Stroke in Women? Data from the Women's Pooling Project. Stroke. 2003; 34: 2812-2816.

8. Peters SAE, Huxley RR, Woodward M. Diabetes as a risk factor for stroke in women compared with men: A systematic review and meta-analysis of 64 cohorts, including 775385 individuals and 12539 strokes. Lancet. 2014; 383: 1973-1980.

9. Giorda $\mathrm{CB}$, Avogaro A, Maggini $\mathrm{M}$, et al. Incidence and risk factors for stroke in type 2 diabetic patients: the DAI study. Stroke. 2007; 38: 1154-1160.

10. Huxley R, Barzi F, Woodward M. Excess risk of fatal coronary heart disease associated with diabetes in men and women: Meta-analysis of 37 prospective cohort studies. BMJ. 2006; 332: 7378.

11. Yusuf S, Hawken S, Ounpuu S, et al. Effect of potentially modifiable risk factors associated with myocardial infarction in 52 countries (the INTERHEART study): case control study. Lancet. 2004; 364: $937-952$.

12. Kissela B, Schneider A, Kleindorfer D, et al. Stroke in a Biracial Population: The Excess Burden of Stroke among Blacks. Stroke. 2004; 35: 426-431.

13. Broderick J, Brott T, Kothari R, et al. The Greater Cincinnati/Northern Kentucky Stroke Study. Stroke. 1998; 29: 415-421.

14. Roy-O'Reilly M, McCullough LD. Sex differences in stroke: the contribution of coagulation. Exp Neurol. 2014; 259: 16-27.

15. Pan A, Wang Y, Talaei M, Hu FB, Wu T. Relation of active, passive, and quitting smoking with incident diabetes: a meta-analysis and systematic review. Lancet Diabetes Endocrinol. 2015; 3: 958-967.

16. Bushnell C, McCullough LD, Awad I a, et al. Guidelines for the prevention of stroke in women: a

This article is protected by copyright. All rights reserved. 
statement for healthcare professionals from the American Heart Association/American Stroke Association. Stroke. 2014; 45: 1545-1588.

17. Stegmayr B, Asplund K. Diabetes as a risk factor for stroke. A population perspective.

Diabetologia. 1995; 38: 1061-1068.

18. Putaala J, Liebkind R, Gordin D, et al. Diabetes mellitus and ischemic stroke in the young: Clinical features and long-term prognosis. Neurology. 2011; 76: 1831-1837.

19. Towfighi A, Ovbiagele B. Metabolic syndrome and stroke. Curr Diab Rep. 2008; 8: 37-41.

Table 1: Sex-Specific Comparison of Stroke Risk Factors in Those With and Without Diabetes (DM)

\begin{tabular}{|lcccc|}
\hline Stroke risk factor (n, \%) & $\begin{array}{c}\text { Women } \\
\text { with } \\
\text { DM } \\
(\mathbf{n = 6 2 7 )}\end{array}$ & $\begin{array}{c}\text { Women } \\
\text { without } \\
\text { DM } \\
(\mathbf{n = 1 2 9 2 )}\end{array}$ & $\begin{array}{c}\text { Men with } \\
\text { DM } \\
\text { (n= 519) }\end{array}$ & $\begin{array}{c}\text { Men } \\
\text { without DM } \\
\text { (n=1077) }\end{array}$ \\
\hline $\begin{array}{l}\text { Age (years) } \\
\text { mean (sd) }\end{array}$ & $70.7(13.4)^{\mathrm{a}}$ & $72.7(15.7)$ & $67.1(13.1)$ & $67.2(14.5)$ \\
\hline $\begin{array}{l}\left.\text { BMI (kg/m }{ }^{2}\right) \\
\text { mean (sd) }\end{array}$ & $30.8(8.0)^{\mathrm{a}}$ & $26.7(6.9)$ & $29.4(6.5)^{\mathrm{b}}$ & $27.0(5.2)$ \\
\hline Age $\geq 65$ years & $427(68.1 \%)$ & $946(73.2 \%)$ & $293(56.4 \%)$ & $621(57.7 \%)$ \\
\hline $\begin{array}{l}\text { Obese } \\
\text { BMI } \geq 30 \text { kg/m }{ }^{2}\end{array}$ & $271(48.7 \%)^{\mathrm{a}}$ & $283(25.3 \%)$ & $201(42.7 \%)^{\mathrm{b}}$ & $209(21.8 \%)$ \\
\hline Black race & $179(28.6 \%)^{\mathrm{a}}$ & $216(16.7 \%)$ & $116(22.4 \%)$ & $186(17.3 \%)$ \\
\hline Hypertension & $574(91.6 \%)^{\mathrm{a}}$ & $950(73.5 \%)$ & $464(89.4 \%)^{\mathrm{b}}$ & $747(69.4 \%)$ \\
\hline High cholesterol & $360(57.4 \%)^{\mathrm{a}}$ & $447(34.6 \%)$ & $313(60.3 \%)^{\mathrm{b}}$ & $442(41.0 \%)$ \\
\hline $\begin{array}{l}\text { Coronary artery disease } \\
\text { (CAD) }\end{array}$ & $253(40.4 \%)^{\mathrm{a}}$ & $299(23.1 \%)$ & $232(44.7 \%)^{\mathrm{b}}$ & $348(32.3 \%)$ \\
\hline $\begin{array}{l}\text { Myocardial infarction } \\
\text { (MI) }\end{array}$ & $97(15.5 \%)^{\mathrm{a}}$ & $114(8.8 \%)$ & $100(19.3 \%)$ & $158(14.7 \%)$ \\
\hline Atrial fibrillation & $159(25.4 \%)$ & $347(26.9 \%)$ & $113(21.8 \%)$ & $220(20.4 \%)$ \\
\hline Current smoker & $113(18.0 \%)$ & $298(23.1 \%)$ & $126(24.3 \%)^{\mathrm{b}}$ & $362(33.6 \%)$ \\
\hline
\end{tabular}

a: $p<0.05$ in bivariate comparison between women with and without DM after Bonferroni correction.

$b: p<0.05$ in bivariate comparison between men with and without DM after Bonferroni correction.

This article is protected by copyright. All rights reserved. 
Table 2: Multivariable analysis by sex of having diabetes according to cardiovascular risk factors among ischemic stroke patients

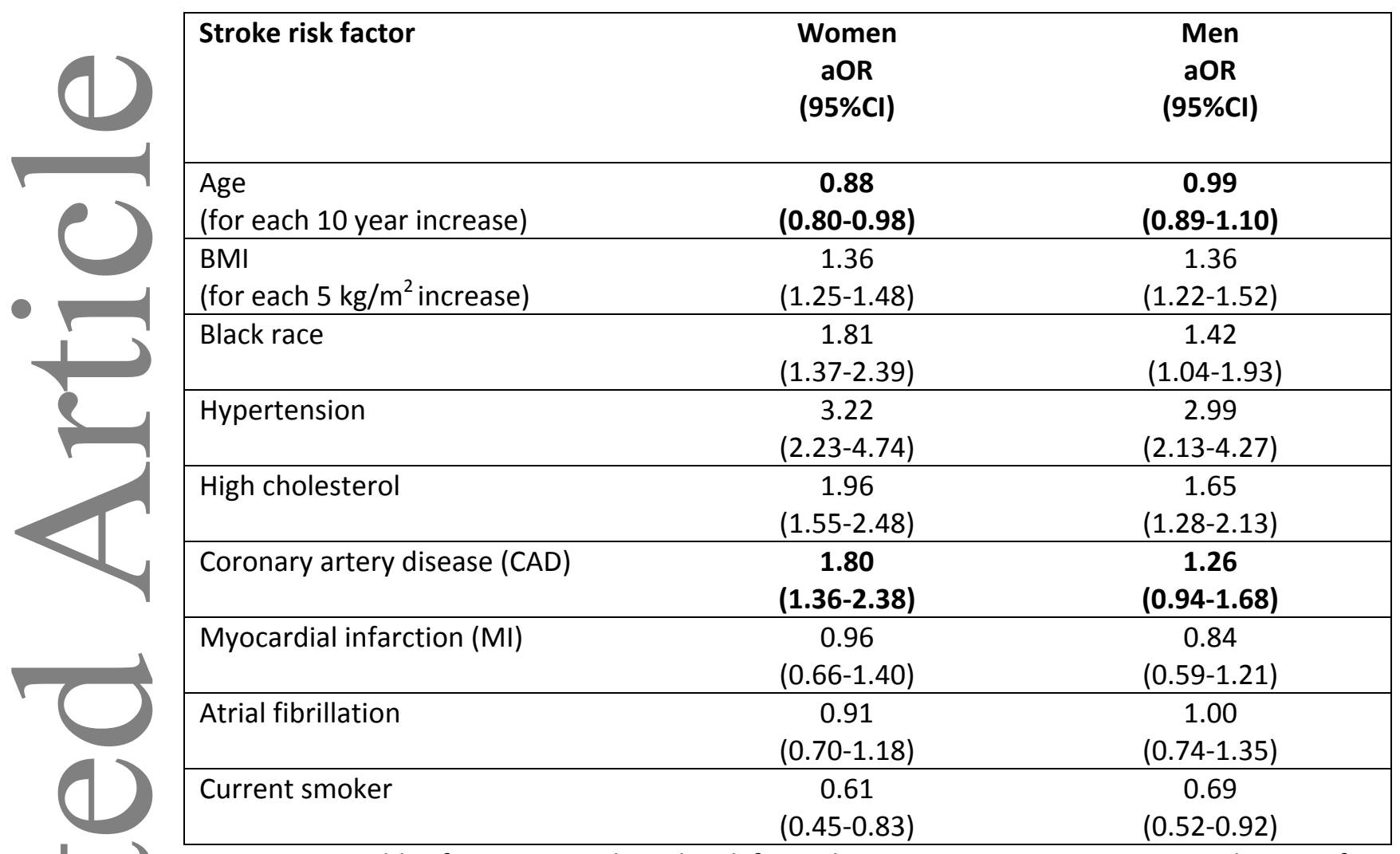

Notes: aOR: Odds of a patient with each risk factor having DM as a pre-existing condition; reference group for each sex is patients without the specified risk factor. Each OR has been adjusted for all other risk factors in table. Bold text indicates that adjusted odds ratios are statistically significant in women but not men.

This article is protected by copyright. All rights reserved. 
Table 3: Medications on Admission by Study Period and Sex Among Adults with Ischemic Stroke in GCNKSS

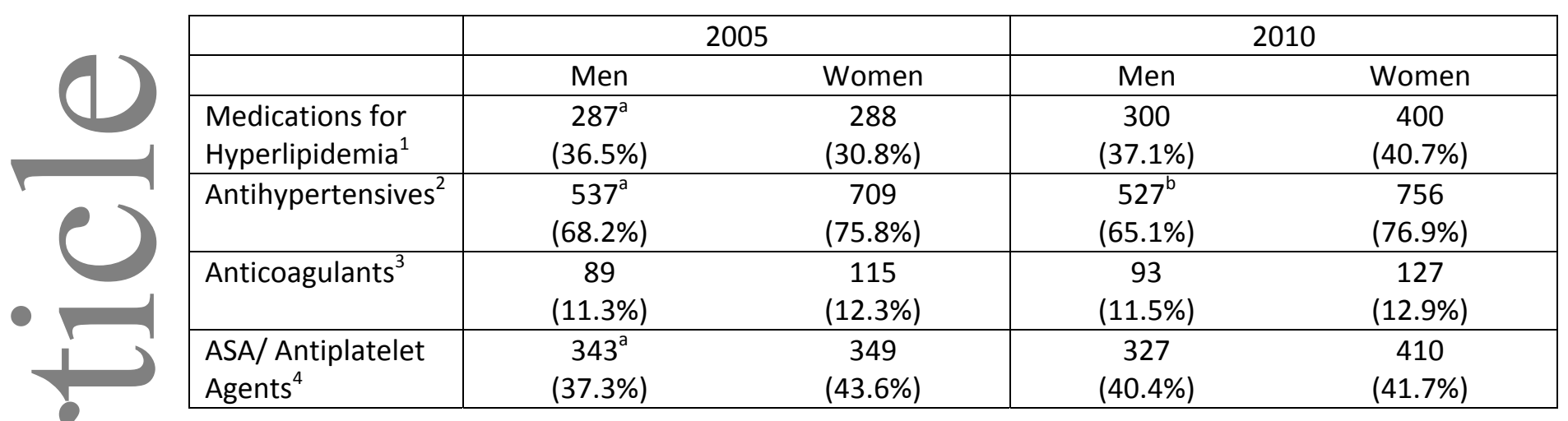

a: $p<0.05$ in bivariate comparison between women and men in 2005.

b: $p<0.05$ in bivariate comparison between women and men in 2010.

1: Medications for hyperlipidemia included statins and other lipid lowering medications. 2:

Antihypertensive medications included ACE inhibitors, alpha 1 blockers, alpha II agonists, angiotensin II receptor blockers, beta blockers, calcium channel blockers, diuretics, vasodilating medications, and nitrates. 3: Anticoagulants included warfarin, heparin, enoxaparin, dalteparin, tinzaparin, and low molecular weight heparin, direct thrombin inhibitors, and factor Xa inhibitors. 4: ASA/ antiplatelet agents included aspirin, clopidogrel, eptifibatide, dipyridamole, cilostazol, ticlopidine, prasugrel, and anagrelide.

This article is protected by copyright. All rights reserved. 